\title{
A white-box model of S-shaped and double S-shaped single- species population growth
}

Lev V. Kalmykov, Vyacheslav L. Kalmykov

Background. A mechanistic modeling of complex systems is one of the main scientific problems today. We consider that this problem may be solved by white-box modeling with using logical deterministic cellular automata. Mathematical models of complex systems are divided into three types: black-box (phenomenological), white-box (mechanistic, based on the first principles) and grey-box (mixtures of phenomenological and mechanistic models). Most basic ecological models are of black-box type, including Malthusian, Verhulst, Lotka-Volterra models. The problem is that the using these black-box models may give us only phenomenological mechanisms, but individual-based (mechanistic) mechanisms of population dynamics remain hidden. Our main goal here is to show possibilities of the white-box modeling in investigating mechanisms of S-shaped and double S-shaped population growth of vegetatively propagated plants. Methods. Using purely logical deterministic individual-based cellular automata we create a white-box model of vegetatively propagated plants. From a general physical standpoint the vegetative propagation of plants is an analogue of excitation propagation in excitable media. A biological prototype of the model is a vegetative propagation of rhizomatous lawn grasses. Using the Monte Carlo method, we investigate a role of different initial positioning of an individual in the habitat. We also investigate different sizes of the habitat, two types of fecundity and two types of boundary conditions. Results. We have created and investigated a basic mechanistic model of one-species population dynamics. This model demonstrates individual-based mechanisms of the S-shaped and double S-shaped population growth. We have investigated mechanisms of the single-species population growth limited by different factors, in particular by resources, habitat size, intraspecific competition, lifetime of individuals, regeneration time and fecundity of individuals. Besides that we have compared the S-shaped and J-shaped population growth. Conclusion. The model demonstrates deterministic individual-based mechanisms of the S-shaped and double S-shaped population growth of vegetatively propagated rhizomatous lawn grasses. An additional important result is a novel demonstration of the white-box modeling of the ecosystem using logical deterministic individual-based cellular automata. We consider this white-box modeling approach as a perspective method of artificial intelligence which works 
as automatic hyper-logical inference from the first principles of the studied subject. This approach may provide direct mechanistic insights into any complex systems. 


\section{A white-box model of S-shaped and double S- 2 shaped single-species population growth}

3 Lev V. Kalmykov ${ }^{1}$, Vyacheslav L. Kalmykov²,3

$4 \quad{ }^{1}$ Institute of Theoretical and Experimental Biophysics, Russian Academy of Sciences, 142290 Pushchino, Moscow

5 Region, Russian Federation

6 ' Institute of Cell Biophysics, Russian Academy of Sciences, 142290 Pushchino, Moscow Region, Russian Federation

7 Pushchino State Institute of Natural Sciences, 142290 Pushchino, Moscow Region, Russian Federation

8 Corresponding author Vyacheslav L. Kalmykov, vyacheslav.l.kalmykov@gmail.com

\section{ABSTRACT}

10 Background. A mechanistic modeling of complex systems is one of the main scientific problems 11 today. We consider that this problem may be solved by white-box modeling with using logical 12 deterministic cellular automata. Mathematical models of complex systems are divided into three 13 types: black-box (phenomenological), white-box (mechanistic, based on the first principles) and 14 grey-box (mixtures of phenomenological and mechanistic models). Most basic ecological models are of black-box type, including Malthusian, Verhulst, Lotka-Volterra models. The problem is that the using these black-box models may give us only phenomenological mechanisms, but individual-based (mechanistic) mechanisms of population dynamics remain hidden. Our main goal here is to show possibilities of the white-box modeling in investigating mechanisms of Sshaped and double S-shaped population growth of vegetatively propagated plants.

Methods. Using purely logical deterministic individual-based cellular automata we create a white-box model of vegetatively propagated plants. From a general physical standpoint the vegetative propagation of plants is an analogue of excitation propagation in excitable media. A biological prototype of the model is a vegetative propagation of rhizomatous lawn grasses. Using the Monte Carlo method, we investigate a role of different initial positioning of an individual in the habitat. We also investigate different sizes of the habitat, two types of fecundity and two types of boundary conditions.

Results. We have created and investigated a basic mechanistic model of one-species population dynamics. This model demonstrates individual-based mechanisms of the S-shaped and double Sshaped population growth. We have investigated mechanisms of the single-species population growth limited by different factors, in particular by resources, habitat size, intraspecific competition, lifetime of individuals, regeneration time and fecundity of individuals. Besides that we have compared the S-shaped and J-shaped population growth.

Conclusion. The model demonstrates deterministic individual-based mechanisms of the S-shaped and double S-shaped population growth of vegetatively propagated rhizomatous lawn grasses. An additional important result is a novel demonstration of the white-box modeling of the ecosystem using logical deterministic individual-based cellular automata. We consider this white-box modeling approach as a perspective method of artificial intelligence which works as automatic 
38 hyper-logical inference from the first principles of the studied subject. This approach may 39 provide direct mechanistic insights into any complex systems.

40 Keywords: population dynamics, complex systems, cellular automata, individual-based

41 modeling, population growth curves, population waves, artificial intelligence

\section{INTRODUCTION}

\section{Background}

44 A mechanistic approach corresponds to the classical ideal of science. Existing mathematical

45 approaches to complex systems modeling are rather phenomenological than mechanistic.

46 Ecologists investigate population dynamics phenomenologically, rather than mechanistically

47 (Tilman 1987). Nonmechanicalness (phenomenologicalness) is still a characteristic for

48 mathematical modeling of complex systems. Most mathematical models in ecology, from simple

49 equations of population growth to complex descriptions of ecosystem dynamics, are not

50 individual-based, i.e. they do not model individuals and their local interactions (Huston et al.

51 1988). The purpose of this study is a mechanistic investigation of the S-shaped and double S-

52 shaped population growth. Individual-based mechanisms of the S-shaped and double S-shaped

53 population growth of vegetatively propagated plants should be completely discrete, logical and

54 consisting of cause-effect and of part-whole relations between micro-subsystems, meso-

55 subsystems and a whole macro-system.

\section{On the types of mathematical models of complex systems}

57 How to create an individual-based mechanistic model of population growth? First, we need to

58 know how to mechanistically model a complex dynamic system. A complex dynamic system may

59 be considered as consisting of interacting subsystems. Interactions between subsystems lead to

60 the emergence of new properties, e.g. of a new pattern formation. Therefore we should define

61 these subsystems and logically describe their interactions in order to create and investigate a

62 mechanistic model. If we want to understand how a complex dynamic system works, we must

63 understand cause-effect relations and part-whole relations in this system. The causes should be

64 sufficient to understand their effects and the parts should be sufficient to understand the whole.

65 There are three types of possible models for complex dynamic systems: black-box, grey-box and

66 white-box models (Fig. 1). 


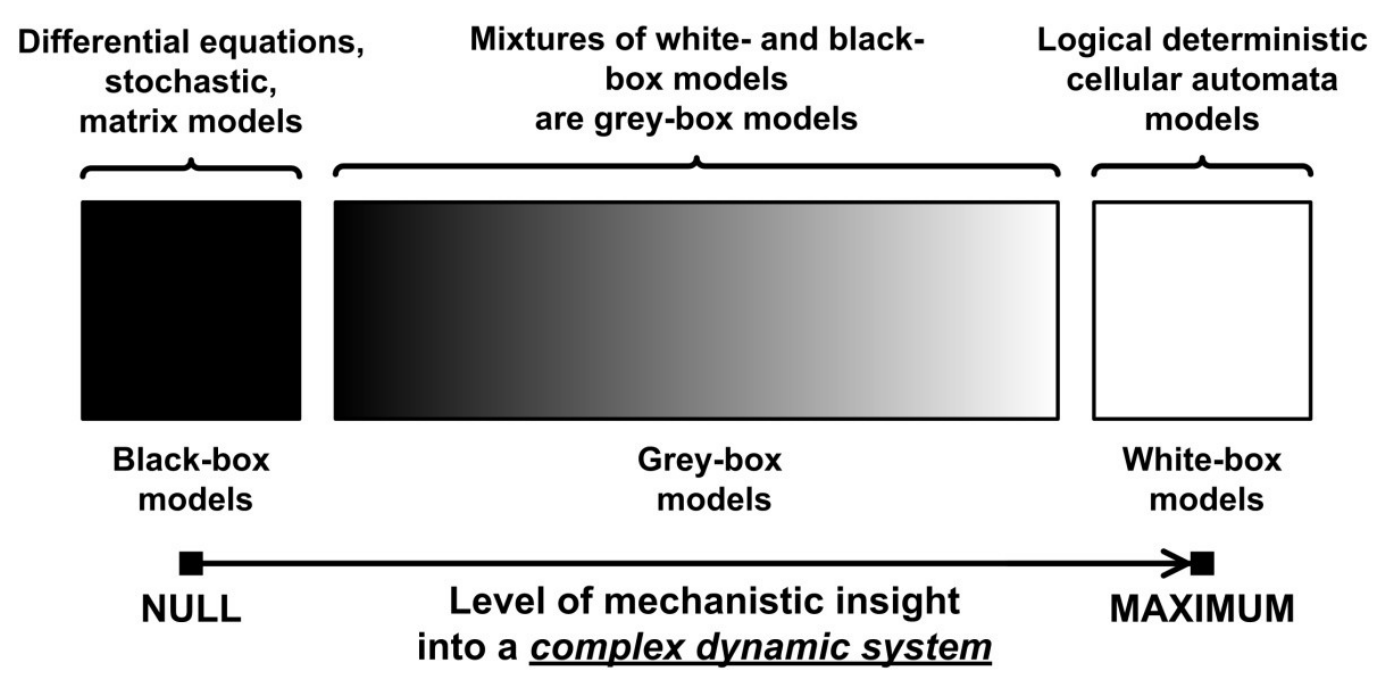

67 Figure 1: Three types of mathematical models for complex dynamic systems. This 68 is a schematic representation of a black-box model, a grey-box model and a white-box 69 model with the level of their mechanistic understanding.

70 Black-box models are completely nonmechanistic. They are phenomenological and ignore a 71 composition and internal structure of a complex system. We cannot investigate interactions of 72 subsystems of such a non-transparent model. A of complex dynamic systems has 'transparent 73 walls' and directly shows underlying mechanisms. All events at micro-, meso- and macro-levels

74 of a dynamic system are directly visible at all stages of its white-box model. Unfortunately, in 75 most cases mathematical modelers prefer to use the heavy black-box mathematical methods, 76 which cannot produce mechanistic models of complex dynamic systems in principle. Grey-box 77 models are intermediate and combine black-box and white-box approaches. As a rule, this 78 approach is used in 'overloaded' form, what makes it less transparent. Basic ecological models 79 are of black-box type, e.g. Malthusian, Verhulst, Lotka-Volterra models. These models are not 80 individual-based and cannot show features of local interactions of individuals of competing 81 species. That is why they principally cannot provide a mechanistic insight into dynamics of 82 ecosystems. Earlier we demonstrated, that the logical deterministic cellular automata approach 83 allows to create the white-box models of ecosystems with interspecific competition between two, 84 three and four grass species (Kalmykov \& Kalmykov 2013). A similar cellular automata model of 85 interspecific competition was created by Silvertown and colleagues (Silvertown et al. 1992). 86 Their model simulates competitive interactions of five grass species, based on experimentally 87 determined rates of invasion. This is a grey-box model as it is based on complex stochastic rules 88 of interspecific interactions. Another similar cellular automata model of single plant species was 89 proposed by Komarov and colleagues, where they represented a link between the concept of 90 discrete description of the ontogenesis of plants and the cellular automata (Komarov et al. 2003).

91 The both two models (Komarov et al. 2003; Silvertown et al. 1992) do not take into account 92 regeneration processes of an ecosystem. 
93 Creation of a white-box model of complex system is associated with the problem of the necessity

94 of an a priori basic knowledge of the modeling subject. The deterministic logical cellular

95 automata are necessary but not sufficient condition of a white-box model. The second necessary

96 prerequisite of a white-box model is the presence of the physical ontology of the object under

97 study. The white-box modeling represents the automatic hyper-logical inference from the first

98 principles because it is completely based on the deterministic logic and axiomatic theory of the

99 subject. The purpose of the white-box modeling is to derive from the basic axioms a more

100 detailed, more concrete mechanistic knowledge about the dynamics of the object under study. We

101 see no other way to obtain a specific and, at the same time, holistic mechanistic understanding of

102 complex systems, apart from the white-box modeling. For providing a strong relevance of our

103 model to the studied problem, we have specified the models' rules (axioms) strictly in accordance

104 with the subject under study. Each logical rule of the model has a correct ecological and physical

105 interpretation. From an ecological point of view we model a vegetative propagation of

106 rhizomatous lawn grasses. From a physical point of view we model propagating of excitation

107 (autowaves, travelling waves, self-sustaining waves) in an excitable (active) medium. The

108 presence of such physical interpretation makes our specific ecological model more general and

109 more natural. The necessity to formulate an intrinsic axiomatic system of the subject before

110 creating its white-box model distinguishes the cellular automata models of white-box type from

111 cellular automata models based on arbitrary logical rules. If cellular automata rules have not been

112 formulated from the first principles of the subject, then such a model may have a weak relevance

113 to the real problem.

\section{On the white-box modeling of population dynamics}

115 Let's consider an example of the inadequacy of some ecological models in result of their

116 incompleteness or incorrectness. There are many models of population dynamics that do not take

117 into account what happens with individuals after their death. Dead individuals instantly disappear

118 with roots, stubs, etc. "One reason for the lack of understanding on the part of most botanists

119 results from their failure to take into account the phenomenon of regeneration in plant

120 communities, which was first discussed in general terms by A. S. Watt in 1947” (Grubb 1977).

121 Stephen Hubbell in his Unified Neutral Theory of Biodiversity (UNTB) in fact refuses a

122 mechanistic understanding of interspecific competition: 'We no longer need better theories of

123 species coexistence; we need better theories for species presence-absence, relative abundance

124 and persistence times in communities that can be confronted with real data. In short, it is long

125 past time for us to get over our myopic preoccupation with coexistence' (Hubbell 2001).

126 However, he admits that 'the real world is not neutral' (Rosindell et al. 2012). Since the basic

127 postulate of the UNTB about ecological neutrality of the similar species in the ecosystem is

128 wrong, this theory cannot be true. In addition, direct local interactions of individuals are absent in

129 the neutral models in principle. That is why neutral models cannot provide a mechanistic insight

130 into biodiversity. The UNTB models are of black-box and dark grey-box types only - Fig. 1. We

131 agree with James Clark, that the dramatic shift in ecological research to focus on neutrality

132 distracts environmentalists from the study of real biodiversity mechanisms and threats (Clark

133 2009). Within the last decade, the neutral theory has become a dominant part of biodiversity 
134 science, emerging as one of the concepts most often tested with field data and evaluated with 135 models (Clark 2009). Neutralists are focused on considering unclear points of the neutral theory 136 the ecological drift, the link between pattern and process, relations of simplicity and complexity

137 in modeling, the role of stochasticity and others, but not the real biodiversity problems

138 themselves (Rosindell et al. 2012). Attempts to understand neutrality instead of biodiversity

139 understanding look like attempts to explain the obscure by the more obscure. Nonmechanistic

140 ecological models make it difficult to answer basic questions, e.g. Why are there so many closely

141 allied species? (Anonymous 1944) An example of the difficult ecological discussion is the

142 debates 'Ecological neutral theory: useful model or statement of ignorance?' on the forum Cell

143 Press Discussions (Craze 2012).Understanding of mechanisms of interspecific coexistence is a

144 global research priority. These mechanisms can allow us to efficiently operate in the field of

145 biodiversity conservation. Obviously, such knowledge must be based on mechanistic models of

146 species coexistence. Unfortunately, ecological modelers prefer to use the heaviest black-box

147 mathematical methods, which cannot produce mechanistic models of complex dynamic systems

148 in principle, and not use simple and long-known purely logical deterministic cellular automata,

149 which can produce white-box models and directly obtain clear mechanistic insights into

150 dynamics of complex systems.

\section{METHODS}

\section{Biological prototype of the model}

153 A vegetative propagation of rhizomatous lawn grasses is the biological prototype of our model

154 (Fig. 3). Festuca rubra trichophylla (Slender creeping red fescue) is the prototype of aggressive 155 vegetative propagation and Poa pratensis L. and Festuca rubra L. ssp. Rubra are the prototypes 156 of moderate vegetative propagation. One individual corresponds to one tiller. A tiller is a minimal 157 semi-autonomous grass shoot that sprouts from the base. Rhizomes are horizontal creeping 158 underground shoots using which plants vegetatively (asexually) propagate. Unlike a root, 159 rhizomes have buds and scaly leaves. One tiller may have maximum three (Fig. 3D) or six 160 rhizoms (Fig. 3B) in the model. Three rhizoms per tiller correspod to moderate propagation (only

161 a half of the nearest microhabitats) and six rhizoms per tiller correspond to aggressive vegetative 162 propagation. A tiller with roots and leaves develops from a bud on the end of the rhizome. A 163 populated microhabitat goes into the regeneration state after an individual's death. The 164 regeneration state of a site corresponds to the regeneration of microhabitat's resources including 165 recycling of a dead individual (Fig. 4). All individuals are identical. Propagation of offsprings of 166 one individual leads to colonization of the uniform, homogeneous and limited habitat (Fig. 2 and 167 Movies S5-S8).

\section{The cellular automata model}

169 We have used logical deterministic individual-based cellular automata to model the S-shaped 170 population growth mechanistically (Fig. 2). This model demonstrates the underlined individual171 based mechanisms. A classical model of the S-shaped population growth is the Verhulst model, 172 which is of completely non mechanistic black-box nature. 
A

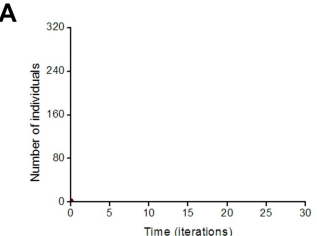

D

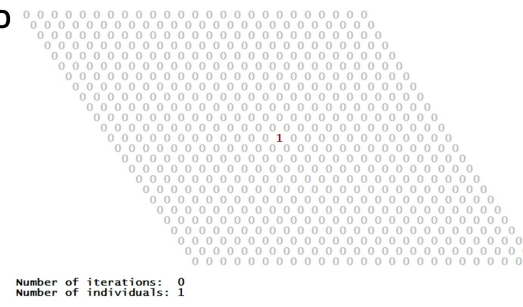

B

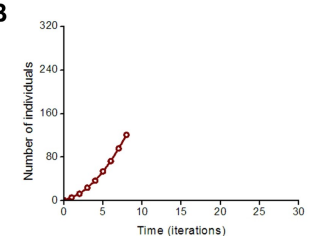

E

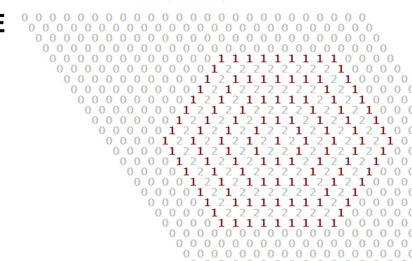

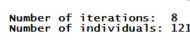

C

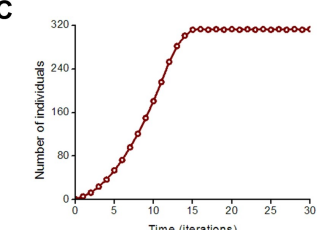

$\mathbf{F}$

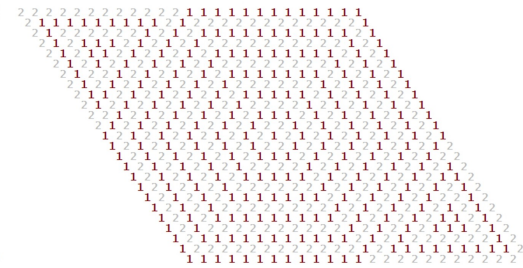

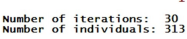

173 Figure 2: S-shaped population growth. A logical deterministic individual-based cellular 174 automata model of an ecosystem with one species shows both population dynamics and 175 pattern formation. The lattice consists of $25 \times 25$ sites. Individuals use the hexagonal 176 neighborhood for their vegetative propagation. The lattice is closed on the torus to avoid 177 boundary effects. (A-C) Population dynamics of the species. S-shaped population 178 growth curve (C). (D-F) Spatio-temporal patterns of the model are represented in 179 numerical form of program implementation.

180 The presented cellular automata model is defined by the 4-tuple:

181

1. a cellular automata lattice, uniting a collection of sites;

2. a finite set of possible states for each lattice site;

184

3. a cellular automata neighborhood which consists of a site and its intrinsically defined neighbors;

4. a function of transitions between the states of a lattice site.

187 The best example of a white-box mechanism is a mechanical watch. Our model metaphorically resembles a mechanical watch in transparent case. A neighborhood logically binds dynamics of all cellular automata sites into one holistic complex dynamic system. There are three most known cellular-automata neighborhoods: von Neumann, Moore and hexagonal. The neighborhood may be of any type. Here we use the hexagonal and tripod neighborhoods which allow to model aggressive and moderate vegetative propagation of rhizomatous lawn grasses (Fig. 3). Different configurations of tripod patterns in Figs 3C and 3D is a result of the fact that the cellular automata neighborhood is implemented successively for each lattice site. 
A

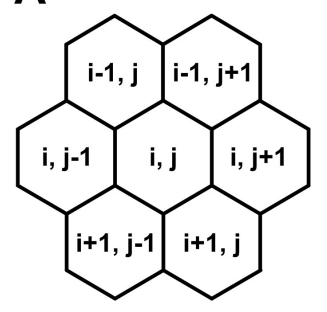

C

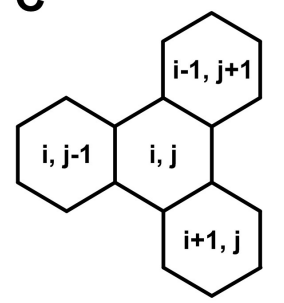

B

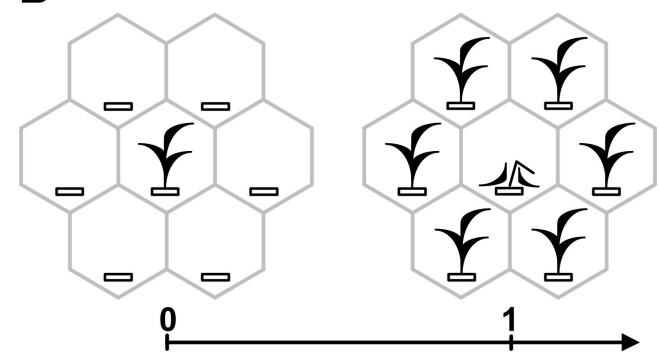

D

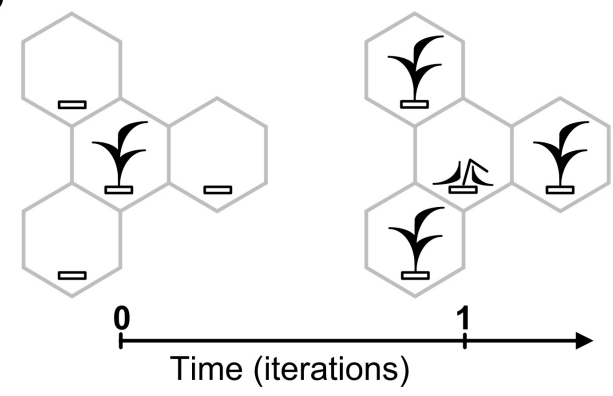

194

195

196

197

198

199

200

201

202

203

204

205

206

207

208

209

210

211

212

213

214

215

216

217

218

219

220

221
Figure 3: Cellular automata neighborhoods. A cellular automata neighborhood models a vegetative propagation of plants and defines fecundity and spatial positioning of an individual's offsprings. Positioning of offsprings is explained by how the cellular automata neighborhood is implemented successively for each lattice site. A central site of the neighborhood is defined by the array element with index $(i, j)$, where $i$ and $j$ are integer numbers. Neighboring sites of the central site are defined by the array elements with indexes. (A) Hexagonal neighborhood. (B) A model example of vegetative propagation of an individual in the hexagonal neighborhood. Offsprings occupy all nearest lattice sites what corresponds to aggressive propagation. A maximum number of offsprings per one individual (fecundity) equals six. (C) Tripod neighborhood. (D) A model example of vegetative propagation of an individual in the tripod neighborhood. Offsprings occupy a half of the nearest lattice sites what corresponds to the moderate propagation. A maximum number of offsprings per one individual equals three.

Integration of reductionist and holistic approaches is one of the challenges for mathematical modeling. Our white-box model of single-species population dynamics opens up new possibilities to solve this challenge. This mechanistic model is hierarchically subdivided into micro-subsystems, meso-subsystems and the whole macro-system. A micro-level is modeled by lattice sites (cellular automata cells). A meso-level of local interactions of micro-objects is modeled by the cellular automata neighborhood. A macro-level is modeled by the entire cellular automata lattice. This is a 'multy-level' modelling as parallel logical operations performed on micro-level, meso-level and macro-level of the model. A unique feature of the cellular-automata is the possibility to model part-whole relationships mechanistically. The relationships of the parts and the whole are modelled using the transition function (combination of the neigbourhood and rules of transition) between states of a lattice site. Parts are the lattice sites and the whole (ecosystem) is the lattice. On each iteration of evolution of the modeled macrosystem the states of its microsystems are changing simultaneously on the basis of logical ruless taking into account states of the neighbouring microsystems (neigbourhood's sites). This allows to model how interactions of microsystems (parts) produce evolution of the macro-system (whole) which leads 
222 to emergence of its new properties (the ecosystem patterns). The white-box cellular automata 223 model shows interactions of parts within the whole, i.e. 'part-whole' relations in the modeled 224 complex system.

225 Figures 3 and 4 illustrate rules of our model.

A

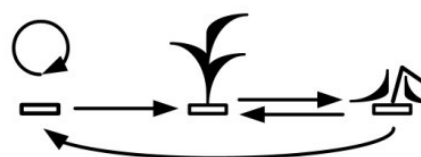

B

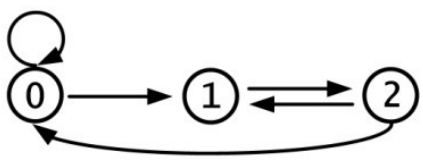

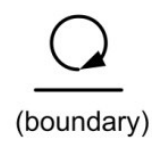

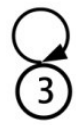

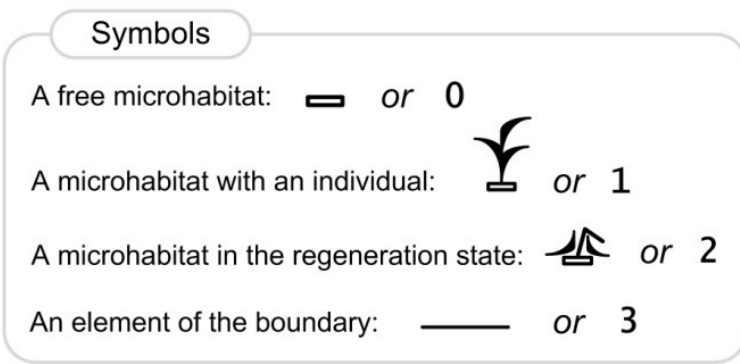

226

227

228

229

230

231

232

233

234

235

236

237

238

239

240

241

242

243

244

245

246

247

248

249

250
Figure 4: Rules of the ecosystem model with one species. Directed graph of transitions between the states of a lattice site is represented in pictorial $(A)$ and numerical forms $(B)$. The graph represents a birth-death-regeneration process.

Here we show a description of the states of a lattice site (microecosystem) in the single species population growth model. Each site may be in one of the four states 0, 1, 2, 3 (Fig. 4), where:

0 - a free microhabitat which can be occupied by an individual of the species;

1 - a microhabitat is occupied by a living individual of the species;

2 - a regeneration state of a microhabitat after death of an individual of the species;

3 - a site in this state represents an element of the boundary that cannot be occupied by an individual.

A free microhabitat is the intrinsic part of environmental resources per one individual and it contains all necessary resources for an individual's life. A microhabitat is modeled by a lattice site.

The cause-effect relations are logical rules of transitions between the states of a lattice site (Fig. 4B):

$0 \rightarrow 0$, a microhabitat remains free if there is no one living individual in its neighborhood;

$0 \rightarrow 1$, a microhabitat will be occupied by an individual of the species if there is at least one individual in its neighborhood;

$1 \rightarrow 2$, after death of an individual of the species its microhabitat goes into the regeneration state;

$2 \rightarrow 0$, after the regeneration state a microhabitat will be free if there is no one living individual in its neighborhood;

$2 \rightarrow 1$, after the regeneration state a microhabitat will be occupied by an individual of the species if there is at least one individual in its neighborhood;

$3 \rightarrow 3$, a site remains in this state, which defines a boundary site.

251 These logical statements are realized for all micro-levels (sites) with their meso-levels

252 (neighborhoods) and thus for the whole macro-level (lattice) of the complex system on each time 
253 iteration. We consider implementation of this algorithm as hyper-logical operations or automatic

254 hyper-logical inference from the first principles of the studied subject.

\section{RESULTS AND DISCUSSION}

256 According to Alexander Watt, a a plant ecosystem may be considered 'as a working mechanism'

257 which 'maintains and regenerates itself' (Watt 1947). Our model demonstrates a such

258 mechanism. From a more general physical point of view we model an active (excitable) media

259 with autowaves (travelling waves, self-sustaining waves) (Kalmykov \& Kalmykov 2013; Krinsky

260 1984; Zaikin \& Zhabotinsky 1970). Active medium is a medium that contains distributed

261 resources for maintenance of autowave propagation. An autowave is a self-organizing and self-

262 sustaining dissipative structure. An active medium may be capable to regenerate its properties

263 after local dissipation of resources. In our model, propagation of individuals occurs in the form of

264 population waves. We use the axiomatic formalism of Wiener and Rosenblueth for modeling of

265 excitation propagation in active media (Wiener \& Rosenblueth 1946). In accordance with this

266 formalism rest, excitation and refractoriness are the three successive states. In our formalism the

267 rest state corresponds to the free state of a microhabitat, the excitation state corresponds to the

268 life activity of an individual in a microhabitat and the refractory state corresponds to the

269 regeneration state of a microhabitat. All states have identical duration. If the refractory period

270 will be much longer than the active period, then such a model may be interpreted, for example, as

271 propagation of the single wave of dry grass fire. Time duration of the basic states can be easily

272 varied using additional states of the lattice sites.

273 Different initial conditions may lead to formation of different spatio-temporal patterns and as a

274 result they may lead to different dynamics of the system. Using the Monte Carlo method, we

275 have investigated the influence of different initial conditions on population dynamics of one

276 species. We have investigated two different boundary conditions, two different cellular automata

277 neighborhoods and four different lattice sizes (Figs 5 and 6). Ecosystem dynamics on the plane

278 with boundary is more natural than on a torus, where boundary effects are absent. The models

279 with non-periodic boundary conditions correspond to laboratory and field experiments where

280 experimental plots also have a boundary. Models with periodic boundary conditions are

281 investigated more commonly, as they allow to avoid boundary effects. Periodic boundary

282 conditions cannot be reproduced in real ecosystems, but they allow to investigate models in a

283 more general form. Therefore, we decided to explore the both types of boundary conditions.

284 Figure 5 shows the results obtained in the study of aggressive propagation and Figure 6 shows the

285 results obtained in the study of moderate propagation. In Figs 5B-D and 6E-H we show the S-

286 shaped population growth and in Fig. 6B-D we show the double S-shaped population growth.

287 Sizes of the lattice which define available space for colonization consisted of $3 \times 3,8 \times 8,23 \times 23$

288 and $98 \times 98$ sites. We have investigated the boundary conditions of two types - when the lattice

289 was closed on the torus by periodic conditions (Fig 5A-D and Fig 6A-D) and when the lattice has

290 a boundary (Fig 5E-H and Fig 6E-H). There were no changes of population dynamics in result of

291 the different initial positioning of an individual on the lattice in cases with periodic boundary

292 conditions (Fig 5A-D and Fig 6A-D). In cases when a lattice has a boundary, different initial 
293 positioning of an individual lead to differences in population dynamics (Fig 5E-H and Fig 6E-H).

294 Moreover, increasing of the lattice may lead to more complex dynamics (Figs 5E-H, 6E-H).

295 Periodic fluctuations in numbers of individuals are observed at the plateau phase in most of the

296 experiments. With increasing of the lattice size, these periodic fluctuations in population size

297 become less visible. The periodic fluctuations on the plateau phase are absent when the lattice

298 consists of $3 \times 3$ sites in the case of the tripod neighborhood (Fig. 6A, 6E). The similar plateau

299 phases without fluctuations were found at the $3 \mathrm{~N} \times 3 \mathrm{~N}$ sizes of the lattice $(6 \times 6,9 \times 9,12 \times 12,15 \times 15$,

$30018 \times 18,27 \times 27$ lattices were tested), with and without boundary effects and when the

301 neighborhood was tripod.

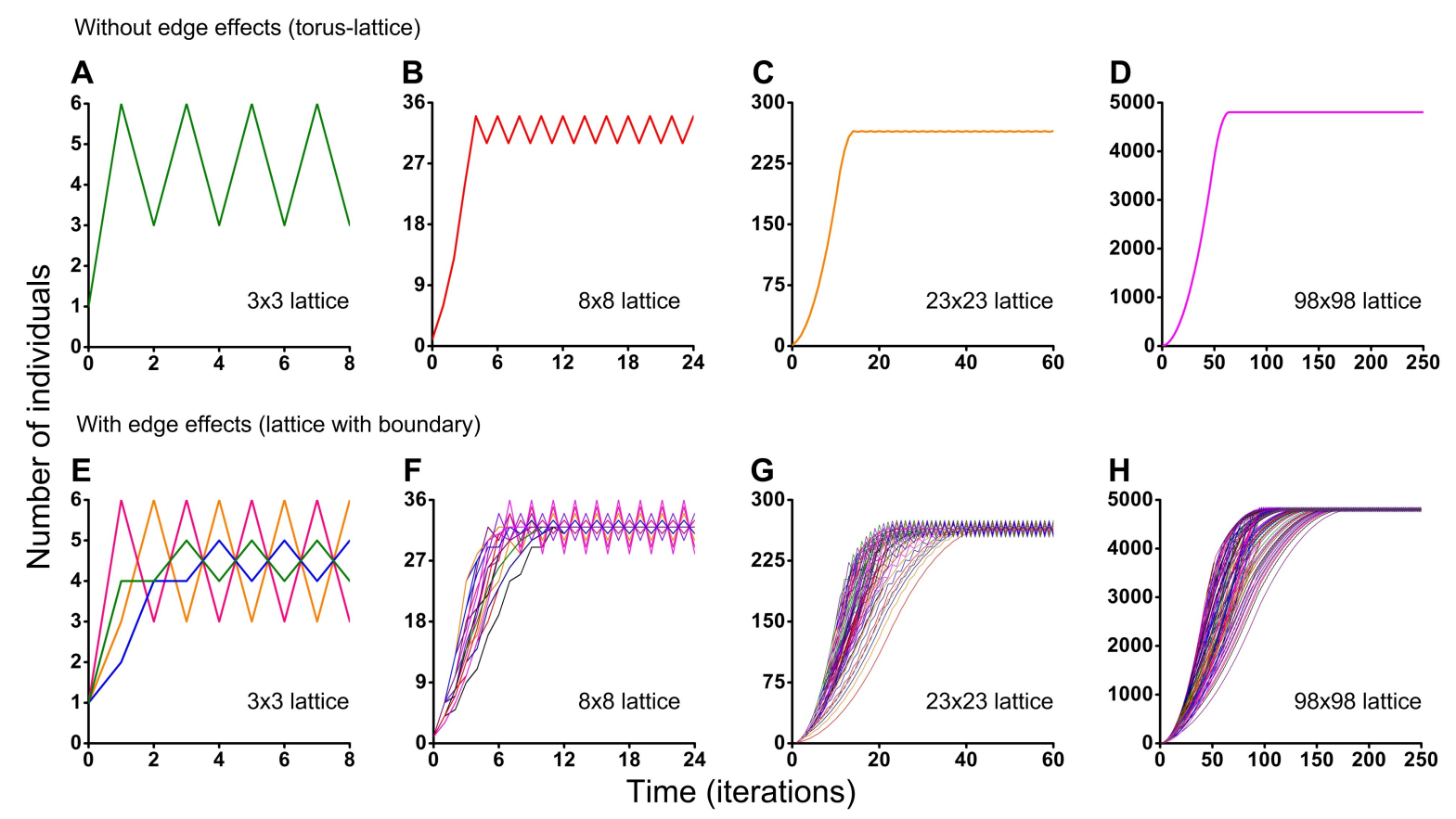

302 Figure 5: Results of the Monte Carlo simulations with the hexagonal

303 neighborhood. Investigation of the influence of boundary conditions, initial positioning

304 of an individual and lattice sizes on single-species population dynamics. (A-D) The

305 lattice is closed on the torus to avoid boundary effects. (E-H) The lattice has a boundary.

306 Every Monte Carlo simulation consisted of 100 repeated experiments with different initial

307 positioning of an individual on the lattice.

308 We show four Movies S1-S4 As examples of the Monte Carlo simulations. Each Monte Carlo 309 simulation consists of five repeated experiments with different initial positioning of an individual 310 on the lattice. The lattices are homogeneous and limited in all experiments. They are consisted of $31123 \times 23$ sites available for occupation by individuals. In Movie S1 the lattice is closed on the torus

312 and the neighborhood is hexagonal. In Movie S2 the lattice has a boundary and the neighborhood 313 is hexagonal. In Movie S3 the lattice is closed on the torus and the neighborhood is tripod. In 314 Movie S4 the lattice has a boundary and the neighborhood is tripod. 

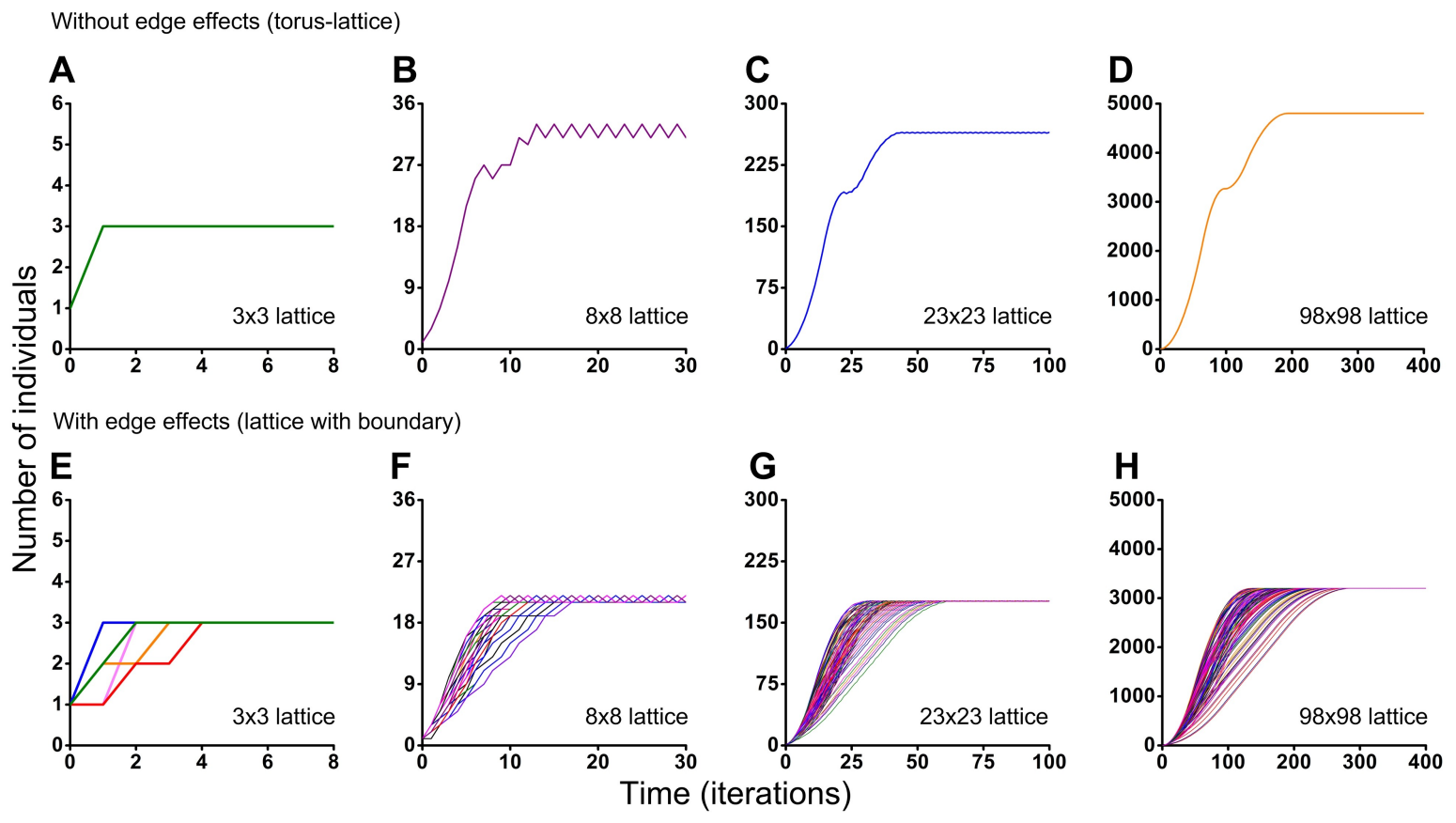

315 Figure 6: Results of the Monte Carlo simulations with the tripod neighborhood.

316 Investigation of the influence of boundary conditions, initial positioning of an individual

317 and lattice sizes on single-species population dynamics. (A-D) The lattice is closed on

318 the torus to avoid boundary effects. (E-H) The lattice has a boundary. Every Monte Carlo

319 simulation consisted of 100 repeated experiments with different initial positioning of an

320 individual on the lattice.

321 In more detail individual-based mechanisms of the double S-shaped population growth curve are 322 presented in Fig. 7C and Movie S7. Details of individual-based mechanisms of three types of the 323 S-shaped population growth curves are presented in Fig. 7A, 7B, 7D and in Movies S5, S6, S8. 


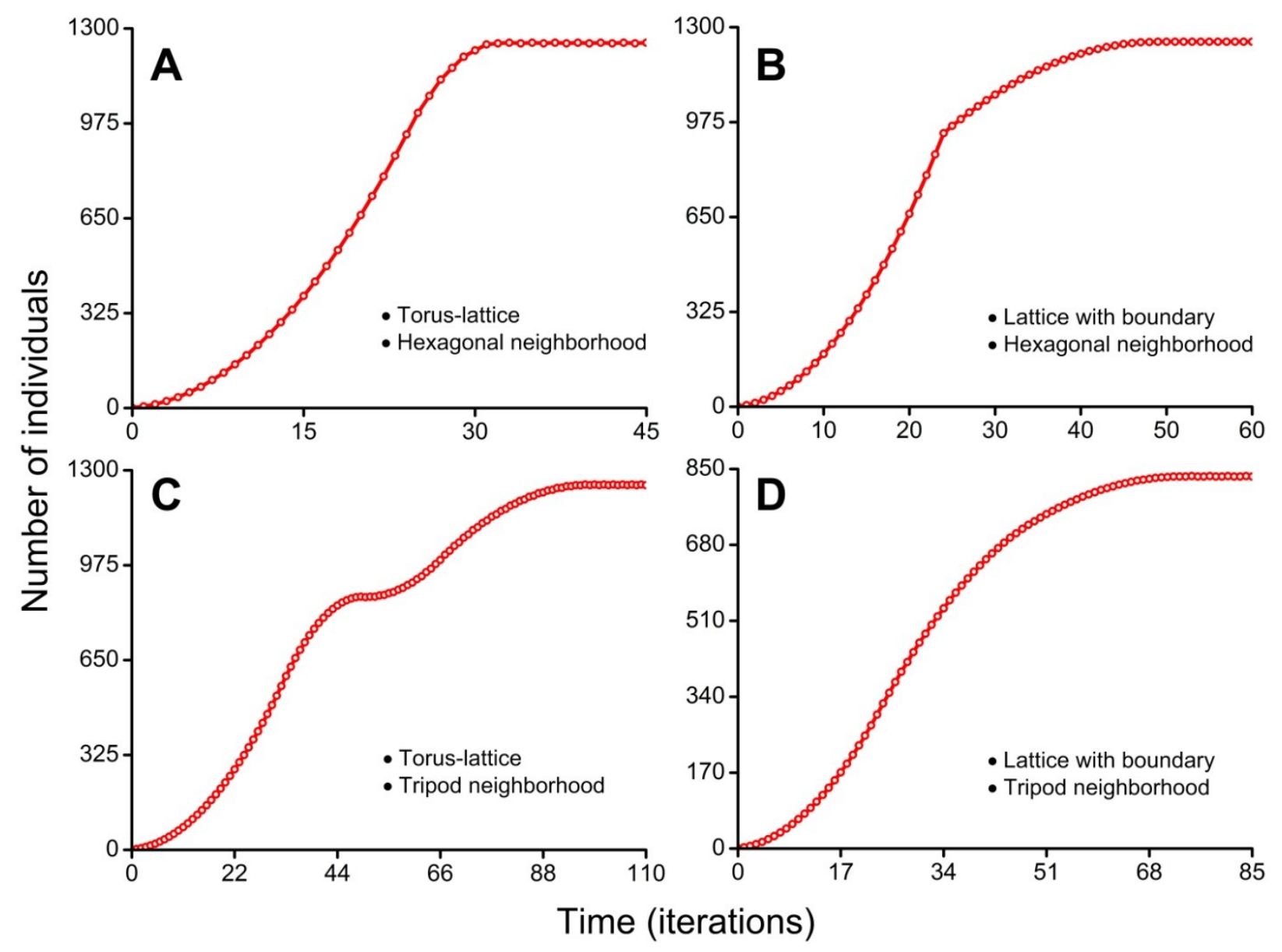

324 Figure 7: Population growth curves. The lattice size which is available for occupation 325 consisted of 50x50 sites in all four cases. (A) S-shaped curve with short phase of

326 decelerating growth. Cellular automata neighborhood is hexagonal and the lattice is 327 closed on the torus (Movie S5). (B) S-shaped curve with sharp transition to long phase

328 of decelerating growth. Cellular automata neighborhood is hexagonal and the lattice has 329 a boundary (Movie S6). (C) Double S-shaped population growth curve. Cellular 330 automata neighborhood is tripod and the lattice is closed on the torus (Movie S7). (D) Sshaped curve with very long phase of decelerating growth. Cellular automata neighborhood is tripod and the lattice has a boundary (Movie S8).

333 Figure 7A shows the S-shaped population growth curve with short phase of decelerating growth. 334 This curve reaches a plateau earlier than on population curves in Figures 7B-D. The plateau is 335 reached on the 32nd iteration (Movie S5). The higher rate of population growth is explained by aggressive propagation and by the lack of boundary effects because the lattice of closed into a

338 Figure 7B shows the S-shaped population growth curve with sharp transition to long phase of 339 decelerating growth. This curve has a sharp slowdown of population growth before the beginning 340 of phase of decelerating growth. It occurs on the 25th iteration, when population waves of 341 aggressively propagating species reach the habitat boundary (Movie S6). In contrast to the curve 342 in Figure 7A, this population curve reaches the plateau on the 49th iteration. Reduced population 
343 growth rate of aggressively propagating species is explained by the presence of boundary effects 344 because the lattice has a boundary.

345 In Figure 7C the population growth curve has a double S-shaped form. The double S-shaped

346 population growth is a result of temporary slowdown of growth, which occurs at the stage when

347 colonization of the free field is replaced by interpenetration of colliding population waves into

348 already occupied areas. Starting from the 34th iteration, the stage of gradually compaction of

349 populated areas begins (Movie S7). This compaction arises from the fact that after rounding of

350 the torus population waves occupy the remaining free sites in the partially populated part of

351 habitat as result of a 'phase shift' of the colliding waves. The free vacancies in population waves

352 remain in result of moderate propagation of individuals. The moderate propagation is modeled by

353 the tripod neighborhood. Speed of the sealing colonization increases slowly due to the form of

354 the population waves which invade into already occupied areas by the expanding wedge. At the

355 same time contribution into population growth from colonization of the areas which consists only

356 of free sites (microhabitats) decreases. The areas which consist only of free microhabitats

357 disappear on the 49th iteration. The population growth rate temporarily slows down what forms

358 the first plateau of the curve. This plateau phase lasts during 5 iterations. The accelerating of

359 additional compactization of population waves leads to the new population growth starting from

360 the 53rd iteration. The population curve reaches the second plateau on the 98th iteration.

361 Figure 7D shows the S-shaped population growth curve with very long phase of decelerating

362 growth. This curve reaches a plateau on the 72nd iteration (Movie S8). Reduced population

363 growth rate and reduced maximum number of individuals in the habitat (834 individuals) are a

364 result of the boundary conditions and the moderate fecundity of individuals (because of tripod

365 neighborhood).

366 The S-shaped and the J-shaped population growth curves

367 We have investigated the S-shaped population growth which is limited by following factors:

368 finite size of the habitat (limited resources), habitats' size, type of boundary conditions of habitat, 369 intraspecific competition, lifetime of individuals, regeneration time of microhabitats, fecundity of

370 individuals (Figs 2, 5-7 and Movies S1-S8). In this section we show the model of the J-shaped

371 population growth and investigate two cases of geometric population growth. Unlike of the S-

372 shaped population model, the J-shaped population model describes a situation in which

373 population growth is not limited in resources, by intraspecific competition or for any other

374 environmental reasons. J-shaped population model describes a full reproductive potential which

375 lead to geometrical population growth (Fig. 8). In other respects this model similar to our model

376 of the S-shaped population growth. It also takes into account natural decline of individuals.

377 Individual's lifetime equals one iteration. 

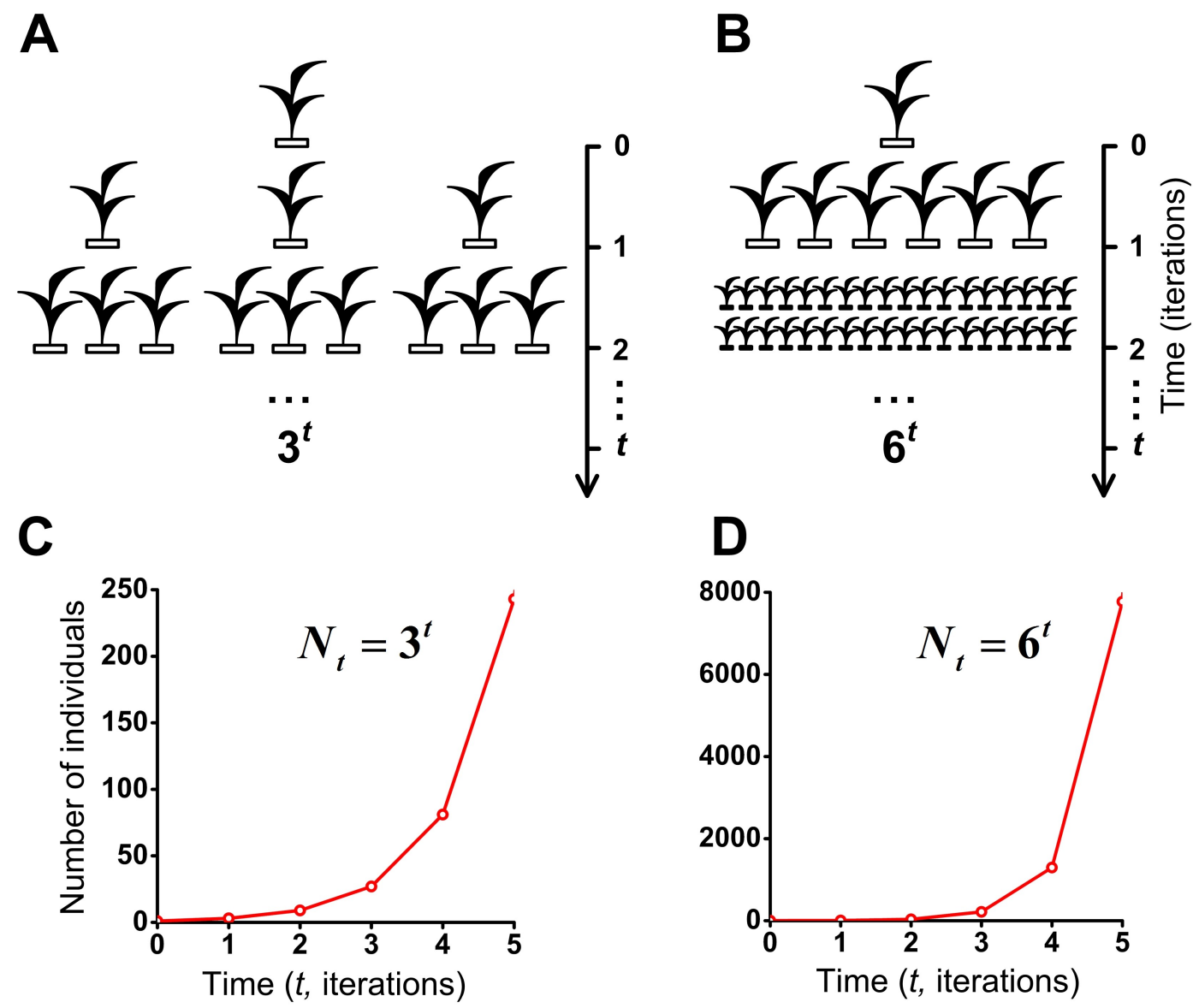

378

379

380

381

382

383

384 385

386

387

388

389

390

391
Figure 8: J-shaped population growth. Propagation of individuals occurs in the absence of intraspecific competition and any restrictions on the resources. A species colonizes an infinite ecosystem under ideal conditions. (A) The number of offsprings per individual equals three. (B) The number of offsprings per individual equals six. (C) Geometric population growth in the first case (A). (D) Geometric population growth in the second case (B).

To assess the effect of intraspecific competition and regeneration of microhabitats on population growth we have compared our model of the S-shaped (Fig. 7A and Movie S5) with the J-shaped population growth (Fig. 8B, D). Comparative dynamics of these models is shown in Table 1. Comparison of these two examples shows that intraspecific competition is a powerful factor which limits population growth. We also compared our double S-shaped population growth model (Fig. 7C and Movie S7) with the J-shaped population growth model (Fig. 8A, C).

Comparative dynamics of these models is shown in Table 2. Thus, we have compared our models of the S-shaped and the double S-shaped population growth with the J-shaped population growth. 
392 Table 1: Comparative population dynamics in the models with the S-shaped and 393 the J-shaped population growth.

\begin{tabular}{|l|l|l|l|l|l|l|}
\hline $\begin{array}{l}\text { Time (Number of iteration and } \\
\text { generation) }\end{array}$ & 0 & 1 & 2 & 3 & 4 & 5 \\
\hline $\begin{array}{l}\text { Number of individuals in the S- } \\
\text { shaped population growth model } \\
\text { (Fig. 7A and Movie S5). Intraspecific } \\
\text { competition exists. Fecundity equals } \\
6 \text { individuals. }\end{array}$ & 1 & 6 & 13 & 24 & 37 & 54 \\
\hline $\begin{array}{l}\text { Number of individuals in the J- } \\
\text { shaped population growth model } \\
\text { (Fig. 8B,D). Intraspecific competition } \\
\text { is absent. Geometric population } \\
\text { growth. Fecundity equals 6. }\end{array}$ & 1 & 6 & 36 & 216 & 1296 & 7776 \\
\hline
\end{tabular}

394 Table 2: Comparative population dynamics in the models with the double S395 shaped and the J-shaped population growth.

\begin{tabular}{|l|l|l|l|l|l|l|}
\hline $\begin{array}{l}\text { Time (Number of iteration and } \\
\text { generation) }\end{array}$ & 0 & 1 & 2 & 3 & 4 & 5 \\
\hline $\begin{array}{l}\text { Number of individuals in the S- } \\
\text { shaped population growth model } \\
\text { (Fig. 7C and Movie S7). Intraspecific } \\
\text { competition exists. Fecundity equals } \\
3 .\end{array}$ & 1 & 3 & 6 & 10 & 15 & 21 \\
\hline $\begin{array}{l}\text { Number of individuals in the J- } \\
\text { shaped population growth model } \\
\text { (Fig. 8A,C). Intraspecific competition } \\
\text { is absent. Geometric population } \\
\text { growth. Fecundity equals 3. }\end{array}$ & 1 & 3 & 9 & 27 & 81 & 243 \\
\hline
\end{tabular}

396 The basic ecological model, which has been presented in this paper, can easily be expanded by 397 the introduction of additional states, different neighborhoods, nested and adjoint lattices 398 (Kalmykov \& Kalmykov 2012). Such extension has allowed us to create pure mechanistic 399 models of interspecific competition between two, three and four species that are complete 400 competitors, and then to verify and reformulate the competitive exlusion principle (Kalmykov \& 401 Kalmykov 2013) in order to solve the biodiversity mystery (Sommer 1999). 


\section{CONCLUSIONS}

403 We have presented and investigated a mechanistic model of dynamics of single species plant 404 population. This model is based on pure logical deterministic individual-based cellular automata. 405 It has a physical and ecological ontology. Here the physical ontology is the ontology of the active 406 medium and ecological ontology represents an ecosytem with one vegetatively propagated plant 407 species. We investigated deterministic individual-based mechanisms underlying the S-shaped and 408 double S-shaped population growth of vegetatively propagated plants. Iimitating modeling of 409 vegetatively propagated rhizomatous lawn grasses was not our main goal. The main goal was 410 demonstration of possibilities of the white-box modeling on example of population growth. The 411 white-box model was made on the basis of physical axioms of excitation propagation in excitable medium. These basic physical axioms of the model have a universal character that, in principle, allows transferring the obtained results to other subject areas. An additional important result is itself demonstration of the white-box modeling of complex systems using logical cellular automata. We consider the details of the "white-box modeling" methodology as the main results of our work. We would like to make this perspective approach more widely used in the practice of mathematical modeling of complex systems. And we have tried to supplement the discussion about "the value of white boxes" by considering specific ways of implementation this model approach. Our study directly introduces the white-box approach into ecological modeling. The white-box approach opens up new perspectives in modeling by implementing a multy-level mechanistic modeling of complex systems. Our deterministic logical cellular automata model works as a system of artificial intelligence. Cellular automata are known as the method of artificial intelligence. But there is a problem how to use this method of artificial intelligence for investigation of complex systems. We show how logical deterministic cellular automata may be used for mathematical white-box modeling of complex systems on example of ecosystem with one species. Parallelism of the logical operations of cellular automata in total volume of the modeled macrosystem allows to speak that the model hyper-logically provides automatic deductive inference. The term 'deductive' is used here because all logical operations are based on axioms. We consider that the main difficulty of this white-box modeling is to create an adequate axiomatic system based on an intrinsic physical ontology of the complex system under study. The main feature of the approach is the use of cellular automata as a way of linking semantics (ontology) and logic of the subject area. Our logical white-box model of an ecosystem with one species combines reductionist and holistic approaches to modeling of complex systems. We consider the white-box modeling by logical deterministic cellular automata as a perspective way for investigation of any complex systems.

436 ACKNOWLEDGEMENTS We thank Kylla M. Benes for helpful suggestions and edits of 437 the earlier version of the manuscript. We would like to thank the Academic Editor Markus

438 Dahlem and the anonymous reviewers for fruitful comments.

439 Supplemental Information Movies S1-S8 can be found online. 


\section{REFERENCES}

441 Anonymous. 1944. British Ecological Society. Easter meeting 1944: Symposium on "The Ecology of Closely Allied

442 Species." Journal of Animal Ecology 13:176-177. doi: http://www.jstor.org/stable/1450?origin=JSTOR-pdf

443 Clark JS. 2009. Beyond neutral science. Trends in Ecology \& Evolution 24:8-15. doi: 10.1016/j.tree.2008.09.004

444 Craze P. 2012. Ecological neutral theory: useful model or statement of ignorance? Cell Press Discussions. URL:

445 http://news.cell.com/discussions/trends-in-ecology-and-evolution/ecological-neutral-theory-useful-model-

$446 \quad$ or-statement-of-ignorance

447 Grubb PJ. 1977. The maintenance of species-richness in plant communities: the importance of the regeneration 448 niche. Biological Reviews 52:107-145. doi: http://dx.doi.org/10.1111/j.1469-185X.1977.tb01347.x

449 Hubbell SP. 2001. The unified neutral theory of biodiversity and biogeography. Princeton, N.J.; Oxford: Princeton $450 \quad$ University Press.

451 Huston M, DeAngelis D, and Post W. 1988. New Computer Models Unify Ecological Theory. Bioscience 38:682-

452 691. URL: http://www.jstor.org/stable/1310870

453 Kalmykov LV, and Kalmykov VL. 2011. Deterministic individual-based cellular automata modelling of single species population dynamics. Available from Nature Precedings:1-15. doi:

$455 \quad$ http://dx.doi.org/10.1038/npre.2011.6661.1

456 Kalmykov LV, and Kalmykov VL. 2012. Mechanistic mechanisms of competition and biodiversity. Available from 457 Nature Precedings:1-34. doi: http://hdl.handle.net/10101/npre.2012.7105.1

458 Kalmykov LV, and Kalmykov VL. 2013. Verification and reformulation of the competitive exclusion principle.

459 Chaos, Solitons \& Fractals 56:124-131. doi: http://dx.doi.org/10.1016/j.chaos.2013.07.006

460 Komarov AS, Palenova MM, and Smirnova OV. 2003. The concept of discrete description of plant ontogenesis and

461

462 cellular automata models of plant populations. Ecological Modelling 170:427-439. doi: http://dx.doi.org/10.1016/S0304-3800(03)00243-6

Krinsky VI. 1984. Autowaves: Results, problems, outlooks. In: Krinsky VI, ed. Autowaves: Results, problems, outlooks in Self-Organization: Autowaves and Structures Far from Equilibrium Berlin: Springer-Verlag, 919.

Rosindell J, Hubbell SP, He F, Harmon LJ, and Etienne RS. 2012. The case for ecological neutral theory. Trends in Ecology \& Evolution 27:203-208. doi: http://dx.doi.org/10.1016/j.tree.2012.01.004

Silvertown J, Holtier S, Johnson J, and Dale P. 1992. Cellular Automaton Models of Interspecific Competition for Space - the Effect of Pattern on Process. Journal of Ecology 80:527-534. URL:

471 Sommer U. 1999. Ecology - Competition and coexistence. Nature 402:366-367. doi: http://dx.doi.org/10.1038/46453

472 Tilman D. 1987. The importance of the mechanisms of interspecific competition. The American Naturalist 129:769-

473 774. doi: http://dx.doi.org/10.1086/284672

474 Watt AS. 1947. Pattern and Process in the Plant Community. Journal of Ecology 35:1-22. doi:

$475 \quad$ http://dx.doi.org/10.2307/2256497 
476 Wiener N, and Rosenblueth A. 1946. The mathematical formulation of the problem of conduction of impulses in a 477 network of connected excitable elements, specifically in cardiac muscle. Arch Inst Cardiol Mex 16:205-265.

478 Zaikin AN, and Zhabotinsky AM. 1970. Concentration Wave Propagation in Two-dimensional Liquid-phase Self479 oscillating System. Nature 225:535-537. doi: http://dx.doi.org/10.1038/225535b0 


\section{1}

Three types of mathematical models for complex dynamic systems.

This is a schematic representation of a black-box model, a grey-box model and a white-box model with the level of their mechanistic understanding.

Differential equations, stochastic, matrix models

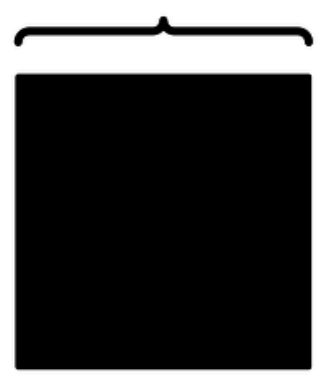

Black-box models
Mixtures of white- and blackbox models are grey-box models

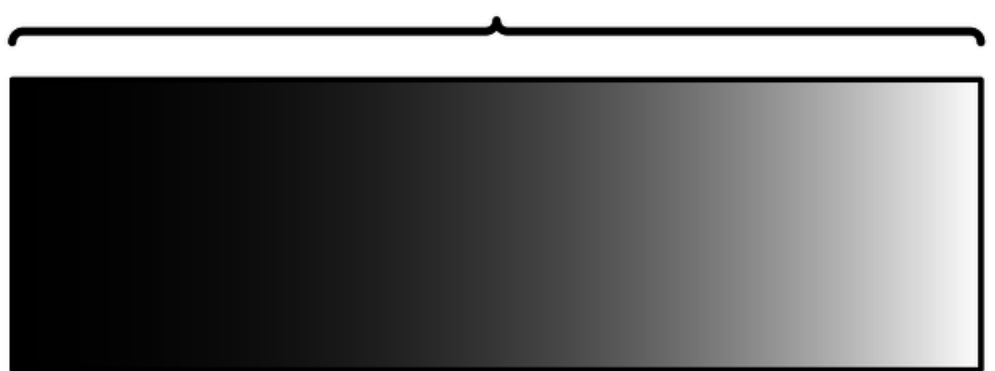

Grey-box

models

Logical deterministic cellular automata models

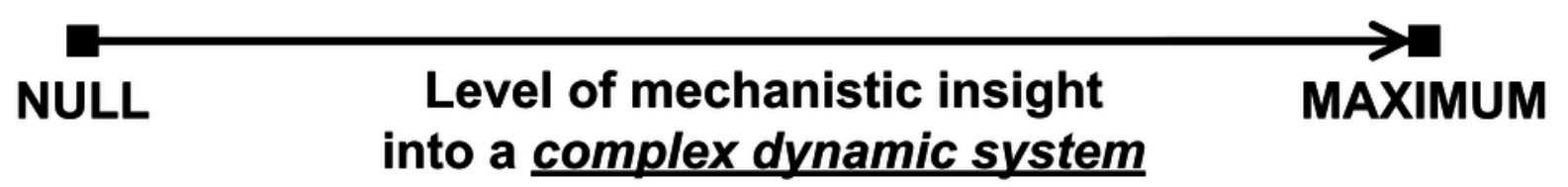


2

S-shaped population growth.

A logical deterministic individual-based cellular automata model of an ecosystem with one species shows both population dynamics and pattern formation. The lattice consists of $25 \times 25$ sites. Individuals use the hexagonal neighborhood for propagation. The lattice is closed on the torus to avoid boundary effects. (A-C) Population dynamics of the species. S-shaped population growth curve (C). (D-F) Spatio-temporal patterns of the model represented in numerical form of program implementation.

A

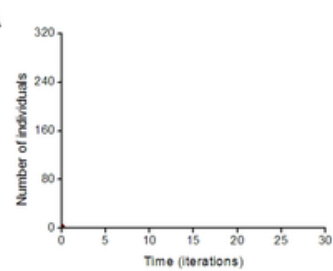

D

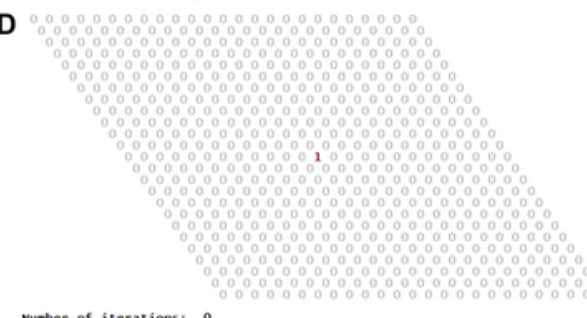

B

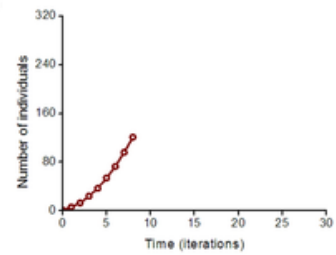

$\mathrm{E}$

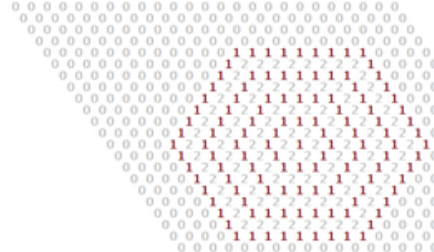

Nusber of iterations: 8 $\mathrm{c}$

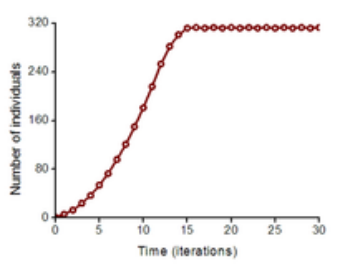

$\mathbf{F}$

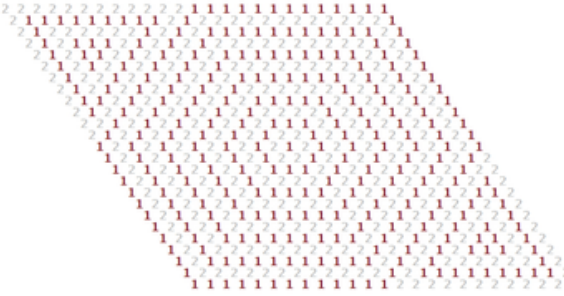

Number of iterations: 30 


\section{3}

Cellular automata neighborhoods.

A cellular automata neighborhood models a vegetative propagation of plants and defines fecundity and spatial positioning of an individual's offsprings. Positioning of offsprings is explained by how the cellular automata neighborhood is implemented successively for each lattice site. A central site of the neighborhood is defined by the array element with index ( $\mathrm{i}$, $j)$, where $i$ and $j$ are integer numbers. Neighboring sites of the central site are defined by the array elements with indexes. (A) Hexagonal neighborhood. (B) A model example of vegetative propagation of an individual in the hexagonal neighborhood. Offsprings occupy all nearest lattice sites what corresponds to aggressive propagation. A maximum number of offsprings per one individual (fecundity) equals six. (C) Tripod neighborhood. (D) A model example of vegetative propagation of an individual in the tripod neighborhood. Offsprings occupy a half of the nearest lattice sites what corresponds to the moderate propagation. A maximum number of offsprings per one individual equals three. 
A

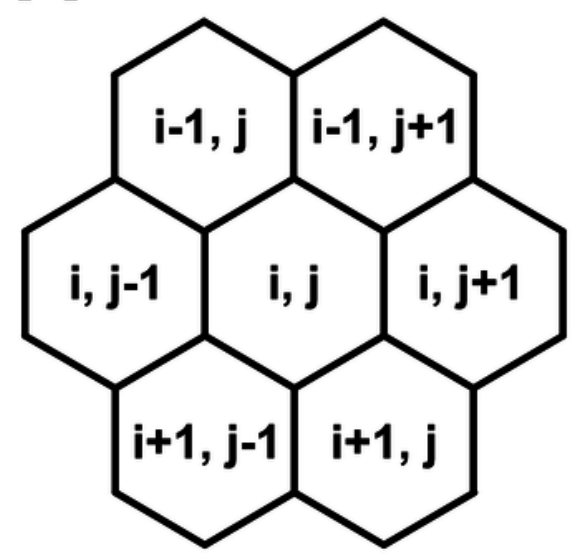

B
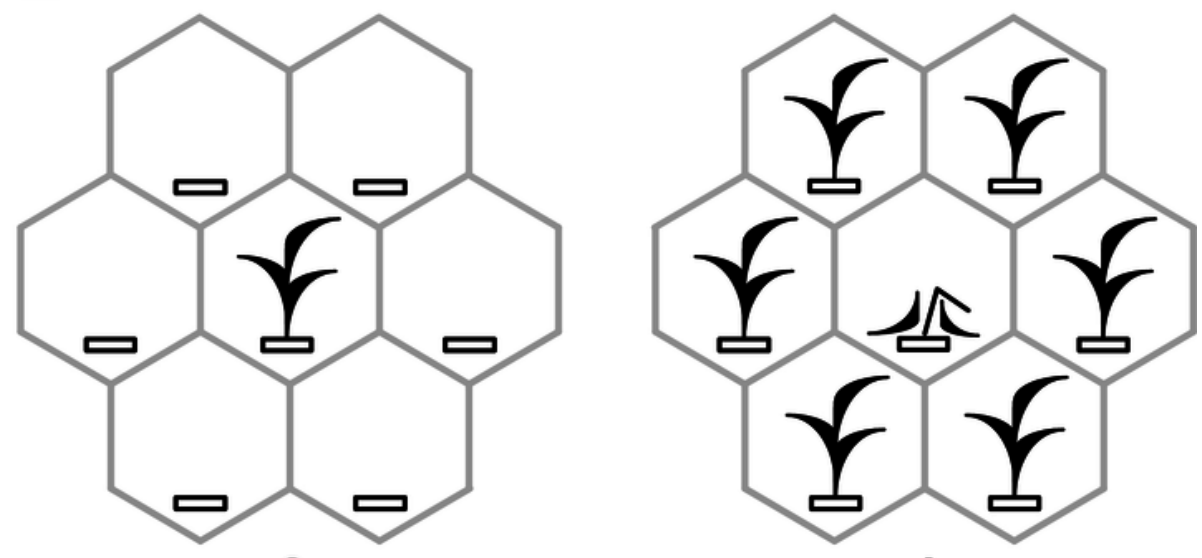

0

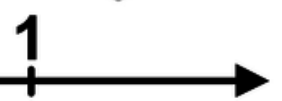

C

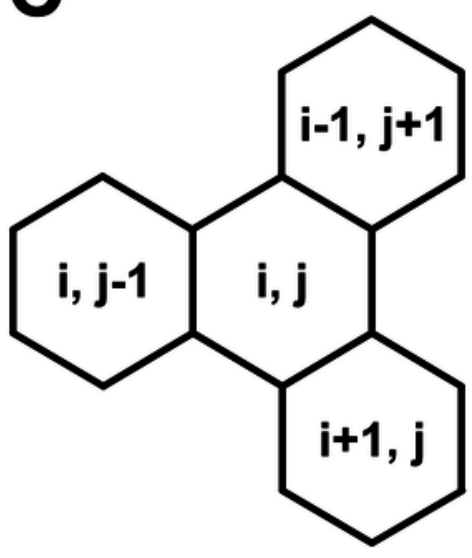

D
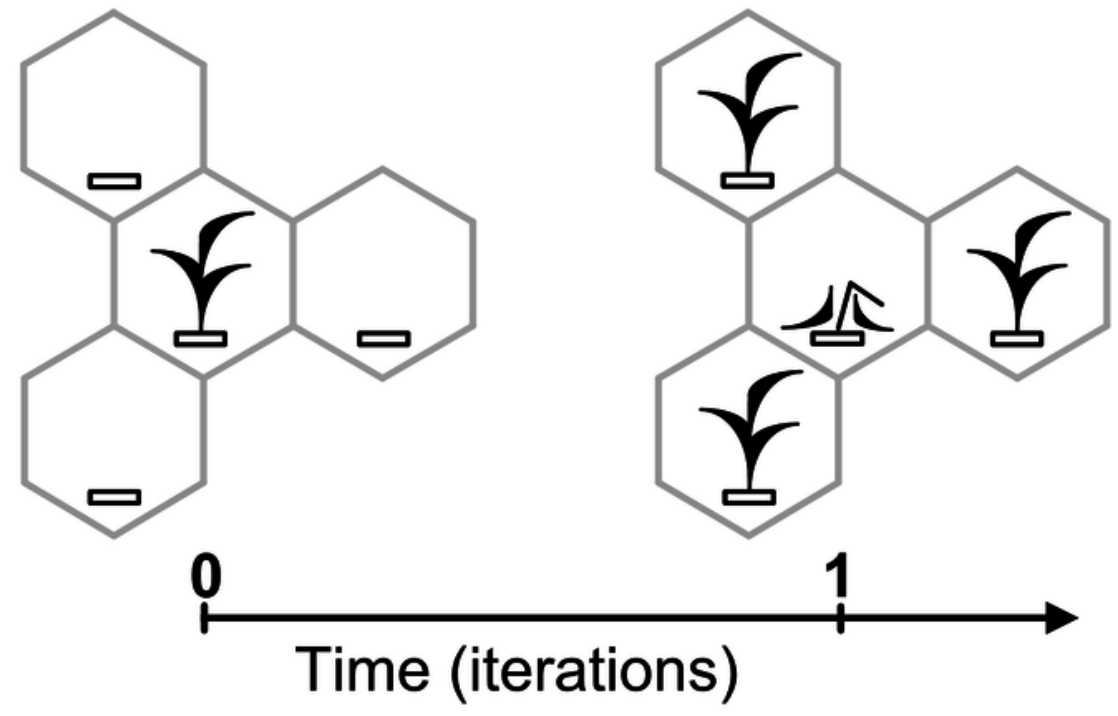
Results of the Monte Carlo simulations with the hexagonal neighborhood.

Investigation of the influence of boundary conditions, initial positioning of an individual and lattice sizes on single-species population dynamics. (A-D) The lattice is closed on the torus to avoid boundary effects. (E-H) The lattice has a boundary. Every Monte Carlo simulation consisted of 100 repeated experiments with different initial positioning of an individual on the lattice.

Without edge effects (torus-lattice)
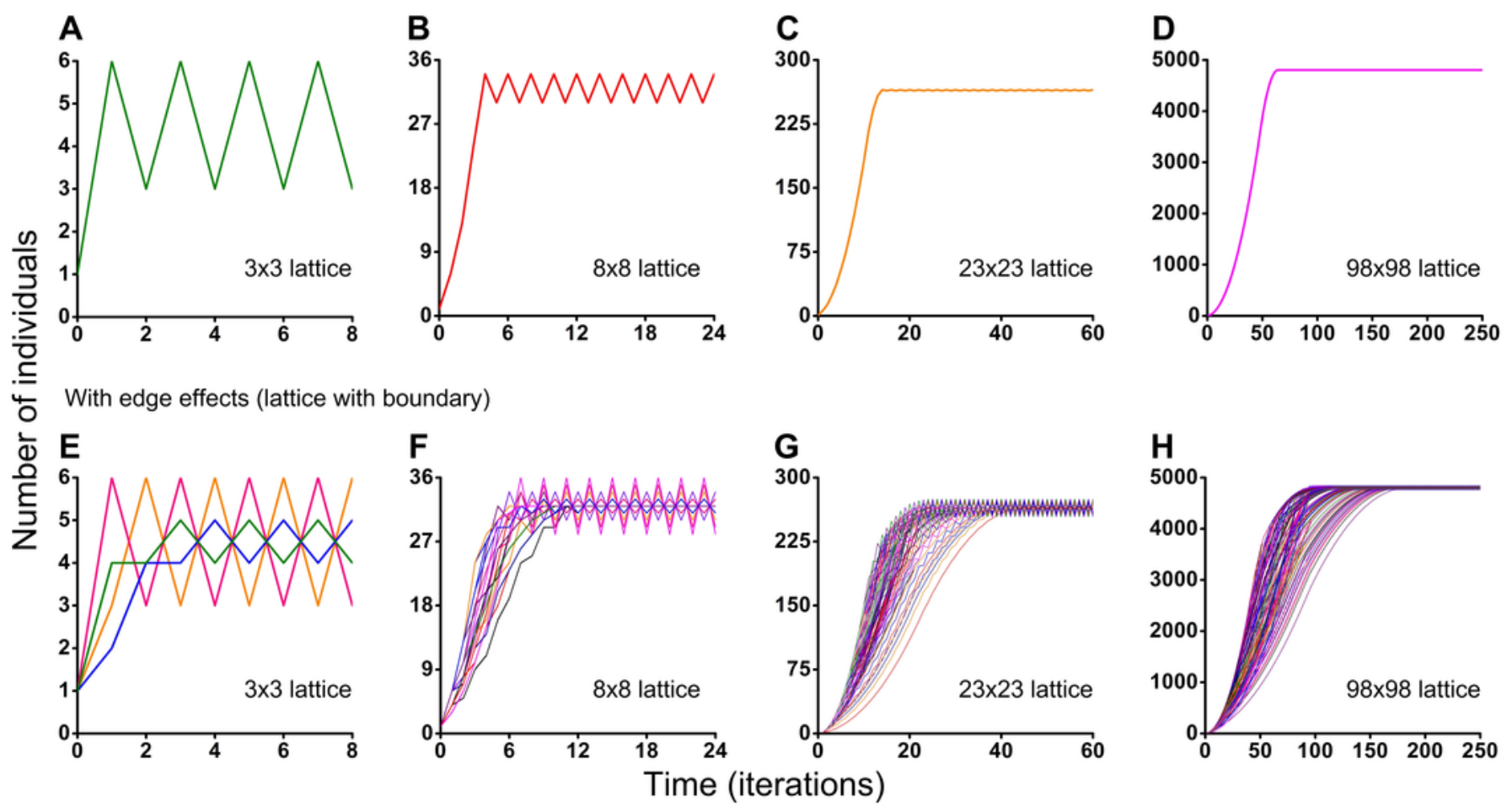


\section{5}

Rules of the ecosystem model with one species.

Directed graph of transitions between the states of a lattice site is represented in pictorial (A) and numerical forms (B). The graph represents a birth-death-regeneration process.

A
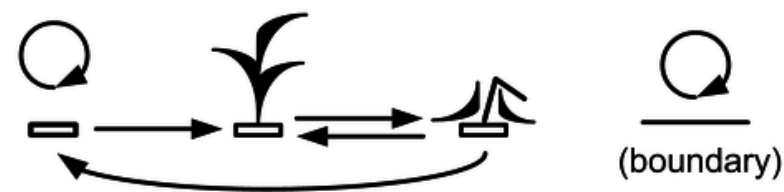

B

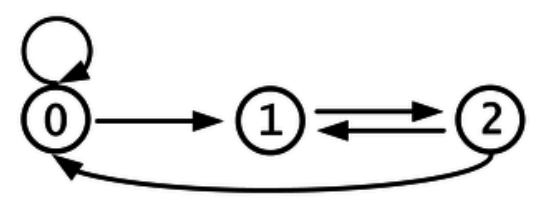

\section{Symbols}

A free microhabitat: $\square$ or 0

A microhabitat with an individual: 5 or 1

A microhabitat in the regeneration state: 垈 or 2

An element of the boundary: $\quad$ or 3 


\section{6}

Results of the Monte Carlo simulations with the tripod neighborhood.

Investigation of the influence of boundary conditions, initial positioning of an individual and lattice sizes on single-species population dynamics. (A-D) The lattice is closed on the torus to avoid boundary effects. (E-H) The lattice has a boundary. Every Monte Carlo simulation consisted of 100 repeated experiments with different initial positioning of an individual on the lattice.

Without edge effects (torus-lattice)
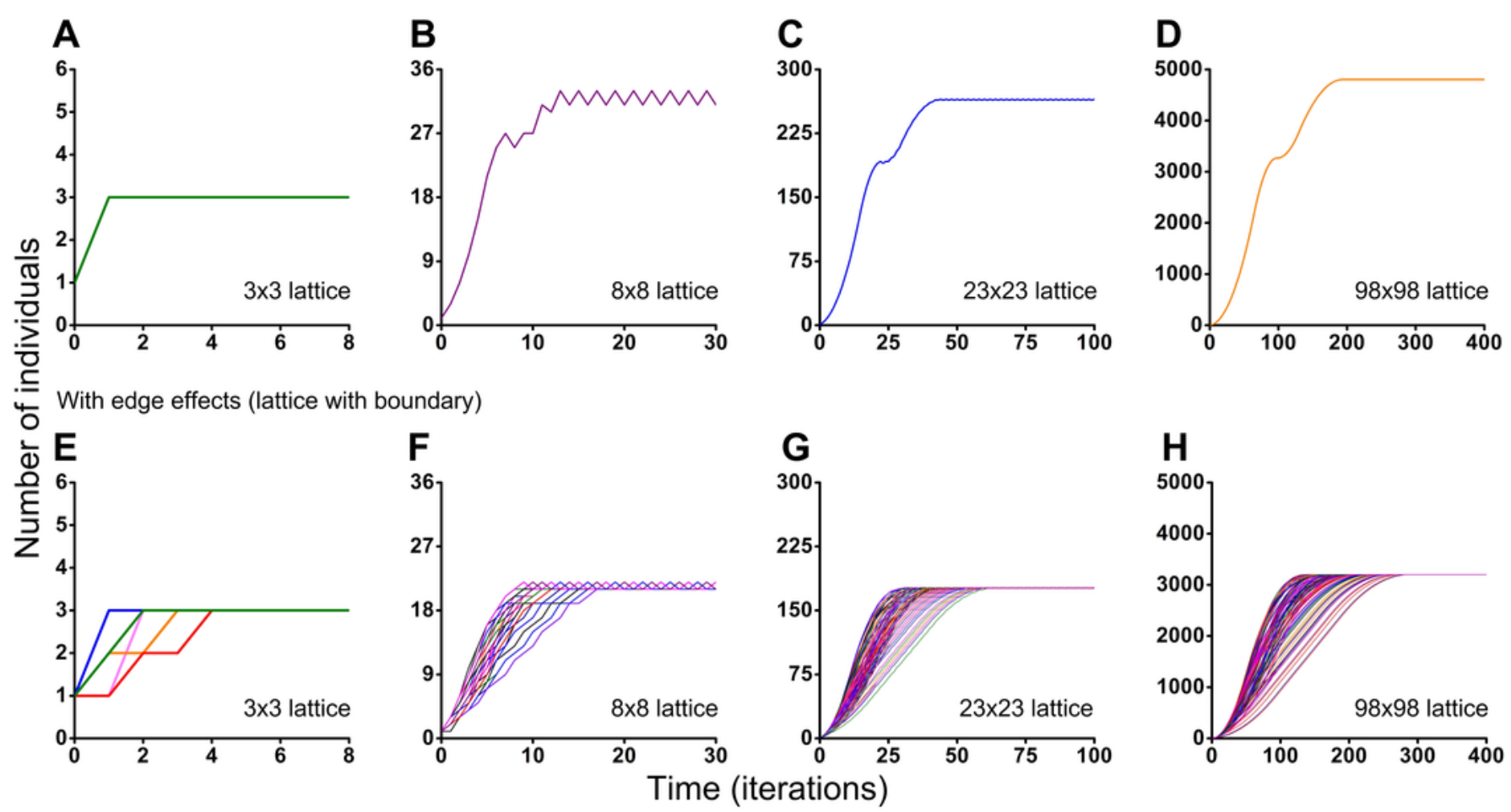


\section{7}

Population growth curves.

The lattice size which is available for occupation consisted of 50x50 sites in all four cases. (A) S-shaped curve with short phase of decelerating growth. Cellular automata neighborhood is hexagonal and the lattice is closed on the torus (Movie S5). (B) S-shaped curve with sharp transition to long phase of decelerating growth. Cellular automata neighborhood is hexagonal and the lattice has a boundary (Movie S6). (C) Double S-shaped population growth curve. Cellular automata neighborhood is tripod and the lattice is closed on the torus (Movie S7). (D) S-shaped curve with very long phase of decelerating growth. Cellular automata neighborhood is tripod and the lattice has a boundary (Movie S8).
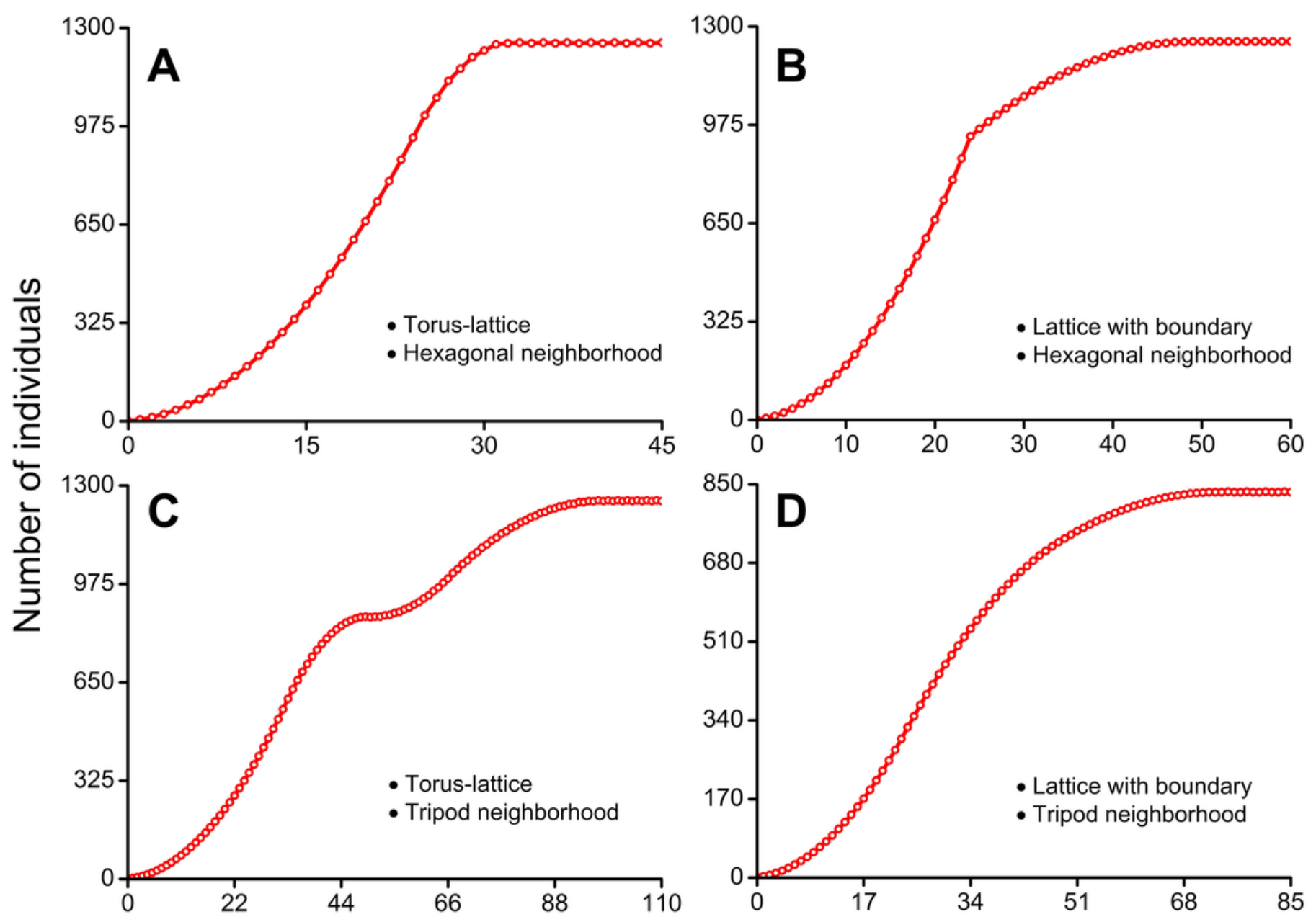
8

J-shaped population growth model.

Propagation of individuals occurs in the absence of intraspecific competition and any restrictions on the resources. A species colonizes an infinite ecosystem under ideal conditions. (A) The number of offsprings per individual equals three. (B) The number of offsprings per individual equals six. (C) Geometric population growth in the first case (A). Geometric population growth in the second case (B).

A

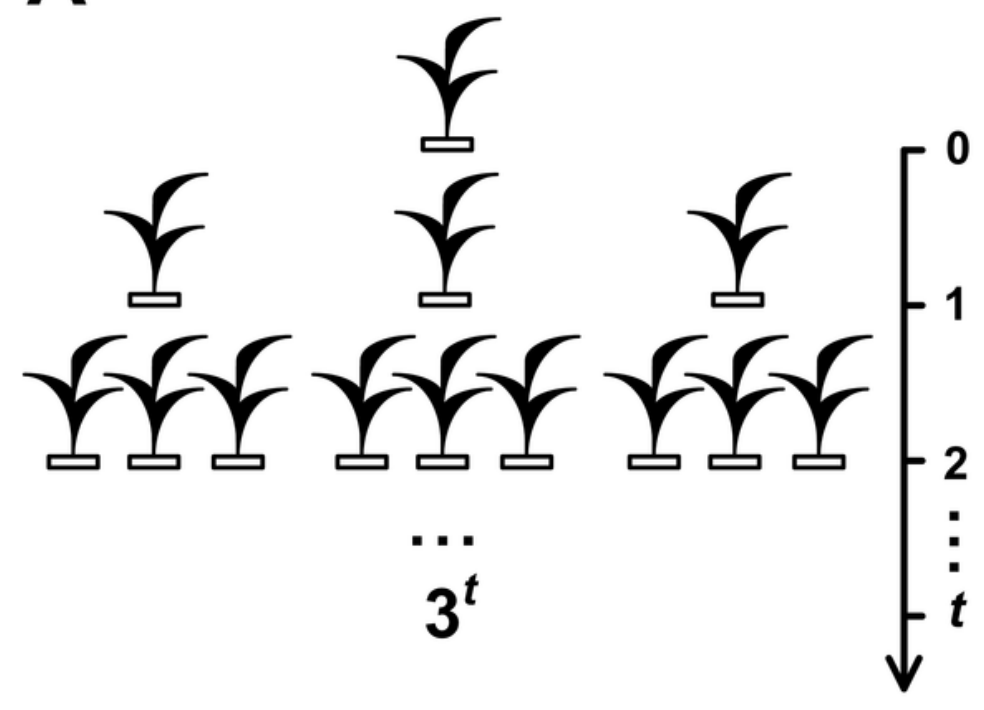

C

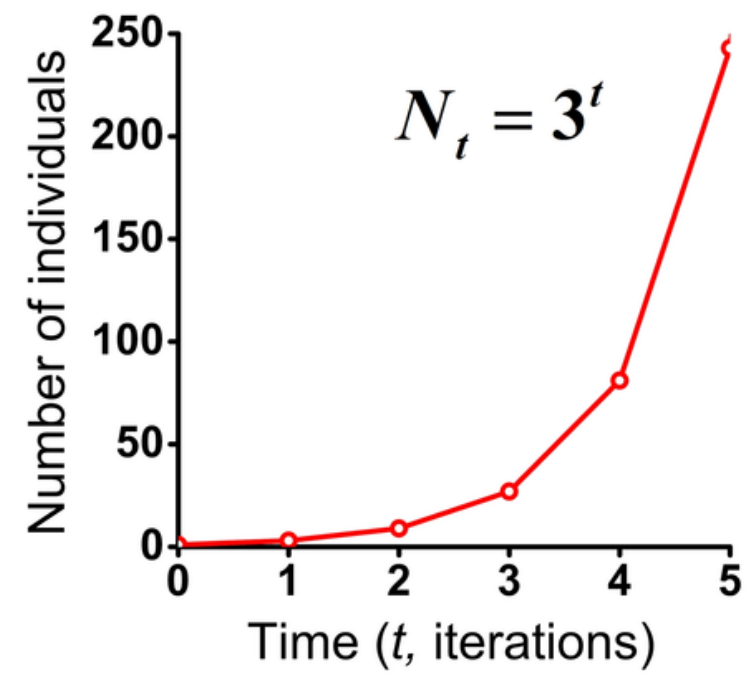

B
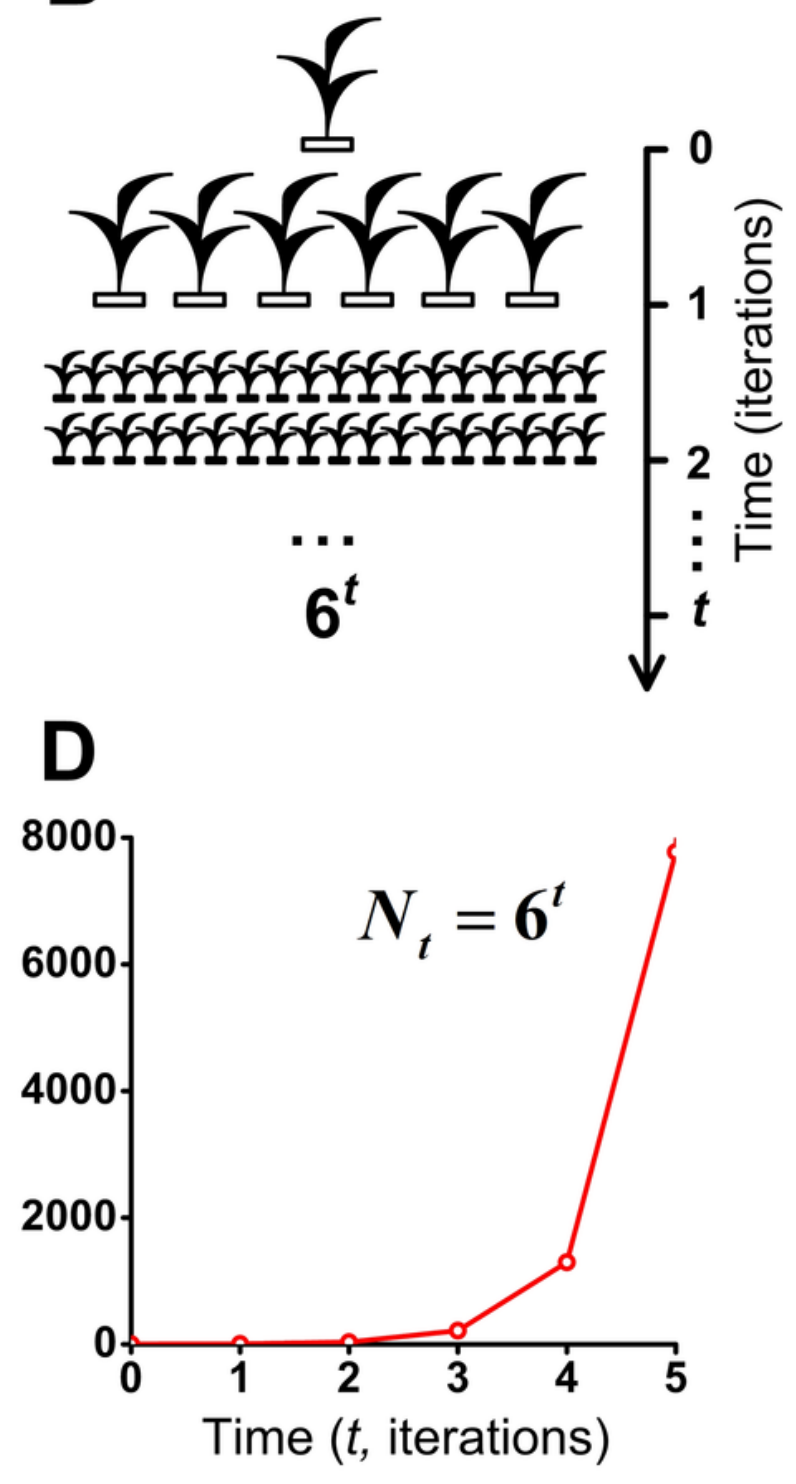


\section{Table $\mathbf{1}$ (on next page)}

Comparative population dynamics in the models with the S-shaped and the J-shaped population growth. 
PeerJ Reviewing Manuscript

\begin{tabular}{|l|c|c|c|c|c|c|}
\hline $\begin{array}{l}\text { Time (Number of iteration and } \\
\text { generation) }\end{array}$ & 0 & 1 & 2 & 3 & 4 & 5 \\
\hline $\begin{array}{l}\text { Number of individuals in the S-shaped } \\
\text { population growth model (Fig. 7A and } \\
\text { Movie S5). Intraspecific competition } \\
\text { exists. Fecundity equals 6 individuals. }\end{array}$ & 1 & 6 & 13 & 24 & 37 & 54 \\
\hline $\begin{array}{l}\text { Number of individuals in the J-shaped } \\
\text { population growth model (Fig. 8B,D). } \\
\text { Intraspecific competition is absent. } \\
\text { Geometric population growth. }\end{array}$ & 1 & 6 & 36 & 216 & 1296 & 7776 \\
Fecundity equals 6.
\end{tabular}


Table 2 (on next page)

Comparative population dynamics in the models with the double S-shaped and the Jshaped population growth. 
PeerJ Reviewing Manuscript

\begin{tabular}{|l|c|c|c|c|c|c|}
\hline $\begin{array}{l}\text { Time (Number of iteration and } \\
\text { generation) }\end{array}$ & 0 & 1 & 2 & 3 & 4 & 5 \\
\hline $\begin{array}{l}\text { Number of individuals in the S-shaped } \\
\text { population growth model (Fig. 7C and } \\
\text { Movie S7). Intraspecific competition } \\
\text { exists. Fecundity equals 3. }\end{array}$ & 1 & 3 & 6 & 10 & 15 & 21 \\
\hline $\begin{array}{l}\text { Number of individuals in the J-shaped } \\
\text { population growth model (Fig. 8A,C). } \\
\text { Intraspecific competition is absent. } \\
\text { Geometric population growth. }\end{array}$ & 1 & 3 & 9 & 27 & 81 & 243 \\
Fecundity equals 3.
\end{tabular}

